\title{
Influence of quantum dot concentration on Förster resonant energy transfer in monodispersed nanocrystal quantum dot monolayers
}

\author{
Manuela Lunz and A. Louise Bradley* \\ Semiconductor Photonics Group, School of Physics, Trinity College Dublin, Dublin 2, Ireland \\ Wei-Yu Chen, Valerie A. Gerard, Stephen J. Byrne, and Yurii K. Gun'ko \\ School of Chemistry, Trinity College Dublin, Dublin 2, Ireland \\ Vladimir Lesnyak and Nikolai Gaponik \\ Physical Chemistry, TU Dresden, Bergstraße 66b, 01062 Dresden, Germany \\ (Received 18 February 2010; revised manuscript received 21 April 2010; published 20 May 2010)
}

\begin{abstract}
The quantum dot (QD) concentration dependence of the optical properties of QD monolayers is shown to be dominated by Förster resonant energy transfer (FRET) from smaller to larger QDs in the ensemble. With increasing QD concentration a redshift of the peak emission wavelength, a shortening of the photoluminescence lifetime of the QDs on the high-energy side of the ensemble emission spectrum as well as increased difference in the lifetimes on the high- and low-energy sides are observed in the layer-by-layer deposited QD monolayers. There is also evidence of an increased rise time in the time-resolved photoluminescence decays on the low-energy side of the QD emission for two of the three samples presented in most detail. A theory of FRET in two dimensions is applied to explain the lifetime decrease on the high-energy side of the ensemble emission and confirms that the impact of the QD concentration on the optical properties is primarily due to FRET from the smaller to larger QDs in the ensemble. The concentration effects are stronger in QD samples which have a broader emission peak compared to the Stokes shift. Based on good agreement with FRET theory, the QD concentration and the overlap of the QD emission and absorption peaks can both be used to control the efficiency of the FRET process in monodispersed QD layers.
\end{abstract}

DOI: 10.1103/PhysRevB.81.205316

PACS number(s): 78.67.Hc, 78.47.jd, 81.16.Dn, 82.20.Rp

\section{INTRODUCTION}

Over the last decades quantum dots (QDs) have attracted a lot of interest in solid-state research due to their unique optical properties. The electron confinement results in very narrow emission and can be easily tuned by changing the size of the QD. ${ }^{1}$ The quality of chemically synthesized colloidal QDs (or nanocrystals) has improved in recent times; due to their high quantum yields and good photostability, colloidal QDs are outperforming molecular dyes in many areas of application. ${ }^{2,3}$ Colloidal QDs are widely used for solution based nanosensors ${ }^{2,4}$ and for biological labeling, ${ }^{5}$ with great advances also reported for optoelectronic devices containing colloidal QDs such as LEDs, ${ }^{6,7}$ photodetectors, and photovoltaics. ${ }^{8-10}$

An additional advantage of colloidal QDs is the possibility for easy and cost-effective processing. QDs can be obtained in different solvents making them suitable for spincoating, drop-casting, or forming Langmuir-Blodgett films. ${ }^{11-13}$ Also the layer-by-layer (LbL) deposition technique, based on the electrostatic self-assembly of oppositely charged macromolecules, ${ }^{14}$ has been shown to be suitable for charged colloidal QDs such as CdTe QDs. ${ }^{15}$ Numerous reports have been published on energy transfer in QD structures formed using these techniques among others. ${ }^{16-23}$ To date there have been only a few reports investigating the influence of the QD concentration on the optical properties and/or energy transfer in monodispersed QD layers, ${ }^{16}$ even though they are the fundamental building blocks of many of the systems and devices highlighted above. It is important to consider the QD concentration when comparing the results reported in different publications; its impact on the signal levels, outcome of measurements and performance of devices has to be determined and taken into account. Additionally, the QD concentration may provide a mechanism for tuning the energy transfer efficiency in QD layers.

The QD monolayers under study were prepared using the LbL technique. It is a fast deposition technique that allows for the formation of closely packed monolayers that are of high interest for applications and that cannot be achieved as easily with the other deposition techniques. Additionally, the $\mathrm{LbL}$ technique enables the realization of complex layered structures at the nanoscale on substrates of any shape and morphology and is therefore a very powerful and flexible tool. ${ }^{12-15}$ Thus, it is used for the preparation of many different types of structures and has, in particular, shown valuable results for the preparation of light emitting and guiding structures, ${ }^{24-27}$ photovoltaic devices, ${ }^{28-30}$ sensors, and detectors. ${ }^{31-33}$ Artificially designed microparticles and nanoparticles with multiple functionality can be synthesized with the help of the LbL technique and have been proposed for applications in areas such as quantum-information processing, optoelectronics, or biotechnology. ${ }^{34-36}$ As the LbL deposition allows for the positioning of layers in nanometer steps, it is also beneficial for the investigation of energy transfer processes $^{17,19,21,22}$ as well as the interaction of surface plasmons with QDs. ${ }^{37-39}$

Previous research reported in the literature has shown that nanocrystal to nanocrystal energy transfer can be appropriately described using Förster resonant energy transfer theory 
despite the inhomogeneous broadening of the QD ensembles and their relatively large size compared to the typical Förster radii of $2-10 \mathrm{~nm}^{40-43}$ Temperature dependence ${ }^{23}$ and distance dependence ${ }^{21,22}$ studies have provided further experimental verification that energy transfer between colloidal nanocrystal QDs occurs by the Förster resonant energy transfer mechanism. Additionally, it was shown that energy transfer in QD systems is occurring resonantly or isoenergetically between the lowest-energy states of smaller QDs, from which the emission arises, and states of larger QDs that correspond to the distinct first absorption peak that is separated from the QD emission feature by the Stokes shift. ${ }^{18}$ We have previously reported on Förster resonant energy transfer (FRET) in mixed QD monolayers consisting of QD donors and acceptors with two different sizes, ${ }^{44}$ and FRET in (mostly three-dimensional) monodispersed QD assemblies has already been observed before. ${ }^{16,18,20,23}$ Here, we present a detailed analysis of FRET in monodispersed QD monolayers giving rise to concentration dependent optical properties.

\section{EXPERIMENTAL METHODS}

Ten different samples of negatively charged, greenemitting colloidal CdTe QDs, stabilized by thioglycolic acid in aqueous solution, were used for the deposition of monolayers on quartz slides by the layer-by-layer electrostatic assembly technique. ${ }^{14,15}$ The QDs were synthesized according to standard procedures. ${ }^{45,46}$ By using a solution with low QD concentration $\left(1.5 \times 10^{-6} \mathrm{M}\right)$ for immersion, the deposited QD layer concentration was varied by changing the immersion times between 1 and $40 \mathrm{~min}$. A graph showing a typical dependence of the QD layer concentration on the immersion time for one of the QD samples can be found in the Appendix.

The QD monolayers were deposited on top of four polyelectrolyte bilayers. The polyelectrolyte layers provide a charged surface for the adsorption of the charged QDs. Each bilayer consists of a negatively charged poly(sodium 4-styrene sulfonate) (PSS) and a positively charged poly(diallyldimethylammonium chloride) (PDDA) layer. PSS and PDDA (20 wt $\%$ solution) were obtained from Sigma Aldrich. The PSS solution for the LbL process was prepared by diluting $560 \mu \mathrm{l}$ of a PSS stock solution (containing $345 \mathrm{mg}$ PSS powder in $10 \mathrm{ml}$ of millipore water) in $5.3 \mathrm{ml}$ millipore water and $0.2 \mathrm{ml}$ of a $3 \mathrm{M} \mathrm{NaCl}$ solution. For the PDDA solution $170 \mu \mathrm{l}$ of the $20 \mathrm{wt} \%$ PDDA solution were mixed with $5.8 \mathrm{ml}$ millipore water and $0.2 \mathrm{ml}$ of the $\mathrm{NaCl}$ solution. The PSS and PDDA bilayers were deposited by immersing the substrate alternating in the PSS and PDDA solutions for $10 \mathrm{~min}$ including an intermediate rinsing step in millipore water for $1 \mathrm{~min}$ between each immersion.

A double beam UV-Vis Recording Spectrometer (Shimadzu UV-2401 PC) was used to measure the absorption spectra of the QD solutions and layers between 350 and 800 $\mathrm{nm}$. Room-temperature steady-state photoluminescence (PL) spectroscopy of the solutions and the layers was performed with a Perkin-Elmer LS 55 fluorescence spectrometer using an excitation wavelength of $400 \mathrm{~nm}$, provided by a pulsed Xenon lamp. The time-resolved PL decays of the QD layers were recorded using a PicoQuant Microtime200 timeresolved confocal microscope system with $150 \mathrm{ps}$ resolution. A LDH-480 laser head, controlled by a PDL-800B driver (PicoQuant), provided picosecond pulses at $470 \mathrm{~nm}$ with an average power of $16 \mathrm{nW}$ and a $10 \mathrm{MHz}$ repetition rate for excitation in these measurements. The PL decays were measured over an area of $80 \times 80 \mu \mathrm{m}^{2}(150 \times 150$ pixels $)$ with an integration time of $4 \mathrm{~ms}$ per pixel. To collect the emission from different parts of the QD ensemble broad band filters centered at 500, 550, and $600 \mathrm{~nm}$ with a full width at half maximum (FWHM) of approximately $(70 \pm 5) \mathrm{nm}$ were used in the PL lifetime measurements. Under these excitation conditions the integrated QD intensity increases linear with pump power, ensuring that not all QDs are excited at the same time and that there is no multiple exciton generation in a single QD.

\section{RESULTS AND DISCUSSION}

Following the presentation of their properties in solution, the concentration dependence of the optical properties of the QD monolayers will be discussed in detail for three representative samples of the ten samples investigated. A theory of FRET in two dimensions ${ }^{47}$ is applied to confirm that the decrease in the lifetime on the high-energy side of the ensemble emission is dominated by FRET from smaller to larger QDs within the ensemble. Furthermore, it is shown that it is sufficient to measure the lifetime difference across the QD ensemble within the monolayer at a single concentration to assess the prevalence of intra-layer FRET. The data for all ten samples follows the trend of the theoretical curve indicating the generality of the results.

\section{A. Optical properties of QD solutions}

In Fig. 1 the PL (squares, left-hand axis) and absorption spectra (lines, right-hand axis) are shown for the solutions of the three samples presented in most detail: QD1, QD2, and QD3. The graphs for these three QD samples will always be represented in the same order: QD1-top panel, QD2center panel, and QD3-bottom panel.

All three samples have PL emission in the green spectral range and a summary of their respective PL emission wavelength, the FWHM of the PL emission spectrum, the position of the first absorption maximum as well as the Stokes shift can be found in Table I. The large FWHM of the PL peak is dominated by the inhomogeneous broadening of the QD ensemble arising from a distribution of QD sizes, shapes, and chemical inhomogeneities present in the sample. ${ }^{1}$ Both, the FWHM and Stokes shift of the QDs presented here lie within the typical range of values observed for colloidal CdTe nanocrystal QDs. QD1 has a large PL FWHM compared to its small Stokes shift, where the Stokes shift is the energy difference between the first absorption peak and the PL emission maximum. The FWHM is almost equal to the Stokes shift for QD2 and is smaller than the Stokes shift for QD3.

The QD size, as well as the concentration of the QDs in solution, can be determined from the position of the first absorption peak as well as the absorption value. ${ }^{48}$ For the 


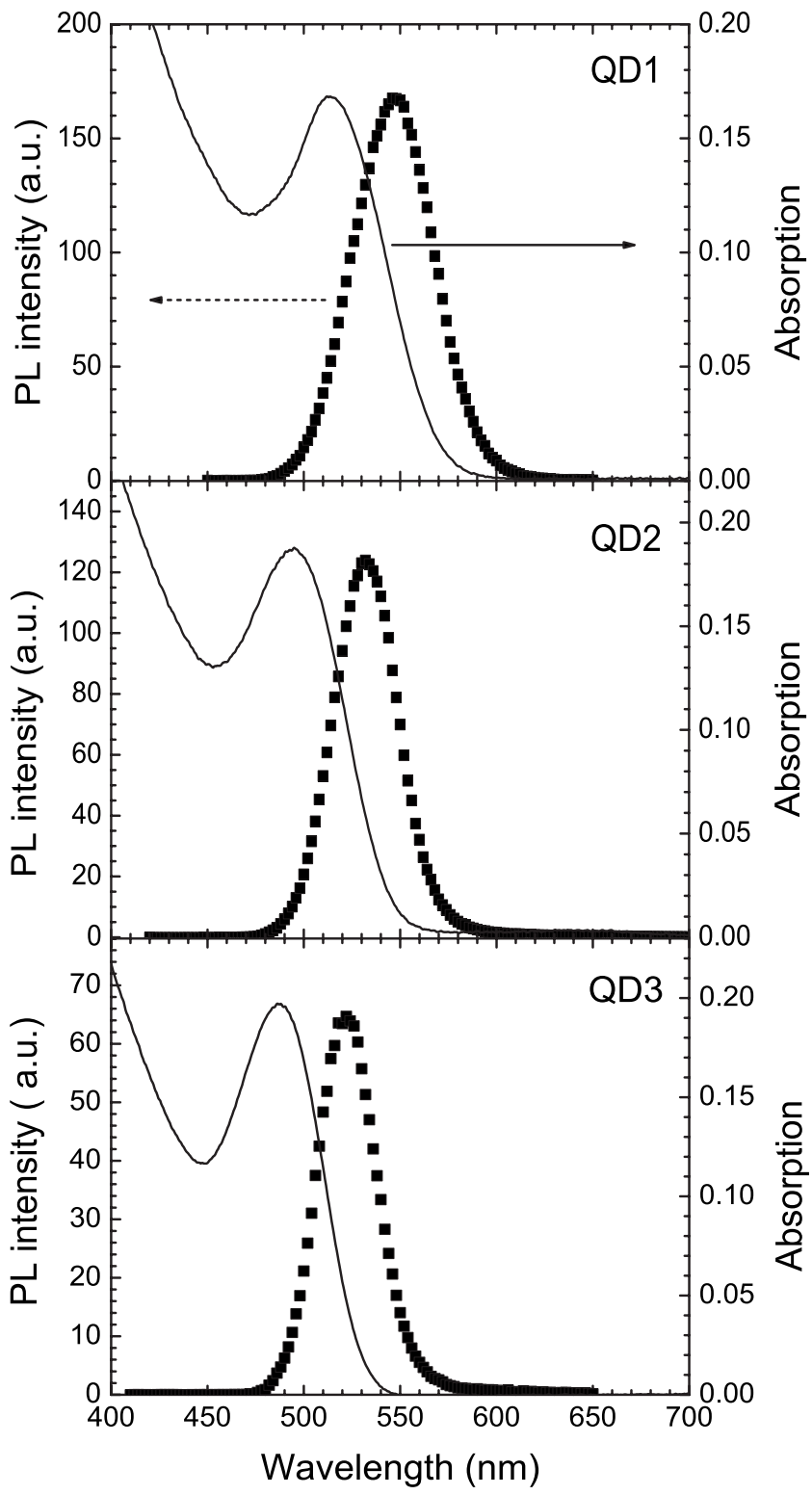

FIG. 1. Emission (solid squares) and absorption spectra (solid lines) of QDs in solution. The top, center, and bottom panels show the spectra of the three samples QD1, QD2, and QD3, respectively.

three QD samples introduced above the QD size is approximately $2.7 \mathrm{~nm}$ in diameter for QD1 and $2.2 \mathrm{~nm}$ for samples $\mathrm{QD} 2$ and QD3. The concentration of the QD monolayers $c_{\mathrm{QD}}$ has been determined by comparing their absorption spectra to the ones of the solutions. Taking into account the change in absorption path length and assuming that the extinction coefficient of the QDs is the same in solution and in the monolayer, the ratio of the peak absorption values is directly proportional to the ratio of the respective concentrations according to Lambert-Beer's law. The concentration $c_{\mathrm{QD}}$ in the QD monolayers was varied between $0.4 \times 10^{17} \mathrm{~m}^{-2}$, corresponding to a QD separation of approximately $5 \mathrm{~nm}$, and $3.5 \times 10^{17} \mathrm{~m}^{-2}$, where closely packed layers are formed. The errors on the concentration varied from maximum $20 \%$ at low concentrations to about $0.2 \times 10^{17} \mathrm{~m}^{-2}$ for concentrations above $1.0 \times 10^{17} \mathrm{~m}^{-2}$

\section{B. Optical properties of QD monolayers}

\section{Observations}

The PL emission of the monolayers had to be corrected for an emission background originating from light reflected by the substrate as well as a weak polyelectrolyte emission. ${ }^{49}$ The integrated QD PL is shown as a function of the QD concentration in the layer in Fig. 2 (solid squares, left-hand axis). The integration was carried out between 450 and 700 $\mathrm{nm}$, and therefore covers the whole emission range of all three QD samples. As expected, the PL intensity increases with increasing QD concentration. For QD1 a linear increase of the intensity with concentration, as indicated by the dashed line, occurs at low concentrations. The slope of the line is proportional to the quantum yield of the QDs in the layers. At higher concentration the PL intensity is increasing at a slower rate, which would correspond to a decrease in the quantum yield. For QD2 and QD3 the PL intensity increases linearly with QD concentration even up to the highest concentrations investigated (Fig. 2). In Fig. 2, the PL peak wavelength for each QD layer is also shown (open circles, righthand axis). It shifts to the red with increasing QD concentration for all three QD samples. For QD1 the emission shifts from about $545 \mathrm{~nm}$ at a concentration of 0.6 $\times 10^{17} \mathrm{~m}^{-2}$ to $561 \mathrm{~nm}$ at $3.4 \times 10^{17} \mathrm{~m}^{-2}$, corresponding to a shift of $16 \mathrm{~nm}$. For QD2 the PL peak shifts by only $9 \mathrm{~nm}$, from 536 to $545 \mathrm{~nm}$, in the same concentration range. No low-concentration data below $1.4 \times 10^{17} \mathrm{~m}^{-2}$ was available for QD3 due to the low quantum yield of this sample and the comparison of the redshift cannot be extended to QD3 in the same detail. Therefore, the peak wavelength shift of all three samples is compared over a smaller concentration range between 1.4 and $3.4 \times 10^{17} \mathrm{~m}^{-2}$ as well. Over this range of concentrations the emission wavelength of the QD3 layers shifts by $4 \mathrm{~nm}$ as is the case for QD2. The QD1 layers again show a larger shift of $6 \mathrm{~nm}$ in this concentration range. A

TABLE I. Optical properties of three QD samples studied; PL peak, PL FWHM, absorption peak, and Stokes shift in wavelength and energy for the QDs in solution.

\begin{tabular}{lcccc}
\hline \hline Sample & $\begin{array}{c}\text { PL peak wavelength/energy } \\
(\mathrm{nm} / \mathrm{eV})\end{array}$ & $\begin{array}{c}\text { PL FWHM } \\
(\mathrm{nm} / \mathrm{meV})\end{array}$ & $\begin{array}{c}\text { First absorption peak } \\
(\mathrm{nm} / \mathrm{eV})\end{array}$ & $\begin{array}{c}\text { Stokes shift } \\
(\mathrm{nm} / \mathrm{meV})\end{array}$ \\
\hline QD1 & $547 / 2.269$ & $49 / 163$ & $514 / 2.412$ & $34.5 / 121$ \\
QD2 & $532 / 2.331$ & $40 / 175$ & $495 / 2.505$ & $37.0 / 174$ \\
QD3 & $522 / 2.375$ & $36 / 164$ & $486.5 / 2.548$ & $35.5 / 170$ \\
\hline \hline
\end{tabular}




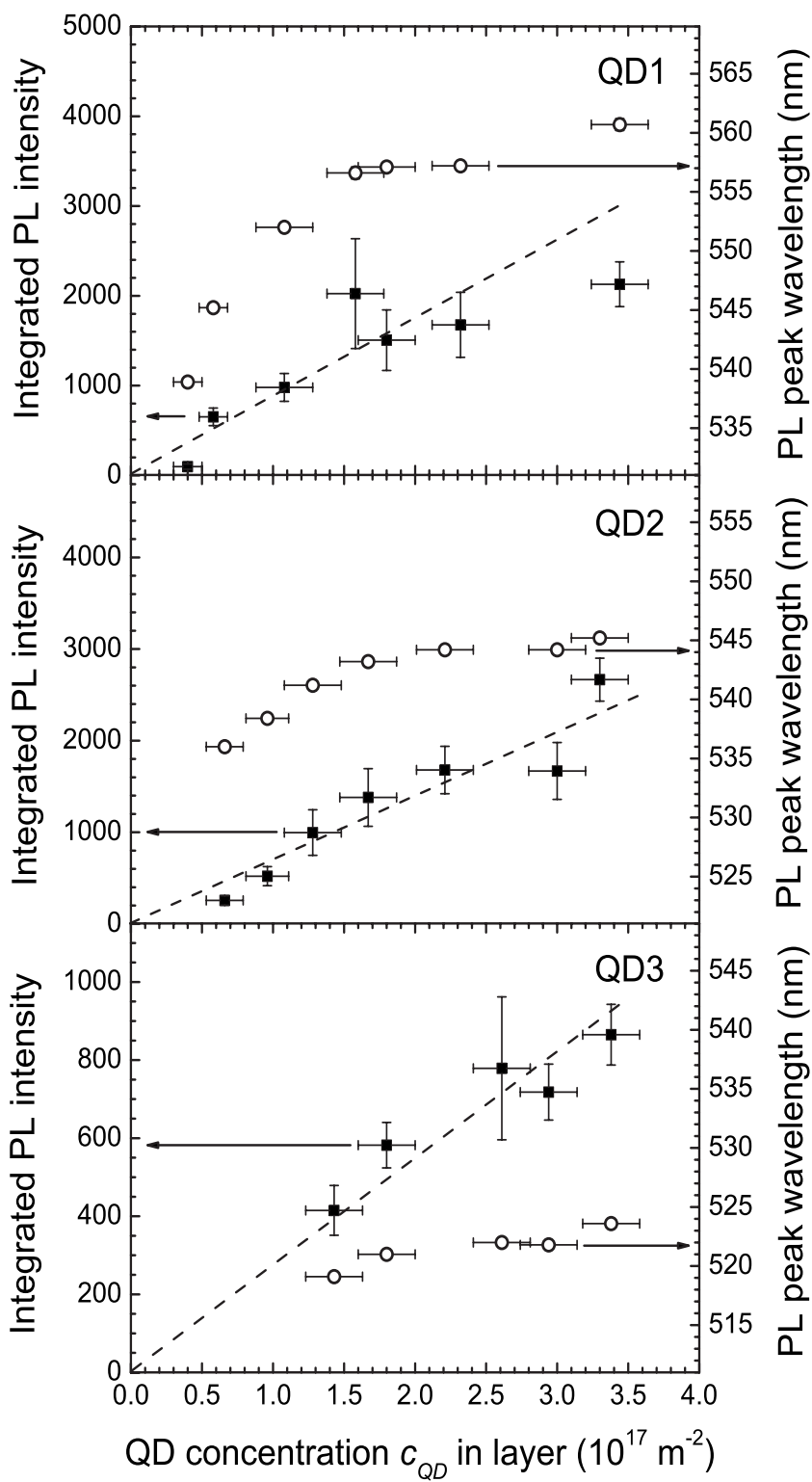

FIG. 2. Dependence of the integrated PL intensity (solid squares, left-hand axis) and the PL peak wavelength (open circles, right-hand axis) on the QD concentration in the monolayers for QD1 (top panel), QD2 (center panel), and QD3 (bottom panel). The dashed lines are included as a guide to the eye to highlight the mostly linear dependence of the integrated PL intensity on the QD concentration. The error on the concentration determined from the monolayer absorption spectra is maximum $20 \%$ at low concentrations and reduces to $0.2 \times 10^{17} \mathrm{~m}^{-2}$ at concentrations higher than $1.0 \times 10^{17} \mathrm{~m}^{-2}$. The error in the determination of the peak wavelength is at most $0.5 \mathrm{~nm}$.

redshift of the PL emission peak is a signature of energy transfer from the blue, high-energy side of the emission spectrum of the QD ensemble (small QDs) to the red, low-energy side (larger QDs). 16,18,20,23

In order to investigate this more closely, time-resolved PL measurements have been carried out on all QD layers. The $\mathrm{PL}$ decays for $\mathrm{QD}$ layers with a concentration $c_{\mathrm{QD}}$ of approximately $2.4 \times 10^{17} \mathrm{~m}^{-2}$ are shown over the first $5 \mathrm{~ns}$ in the three panels of Fig. 3 for the three QD samples. The

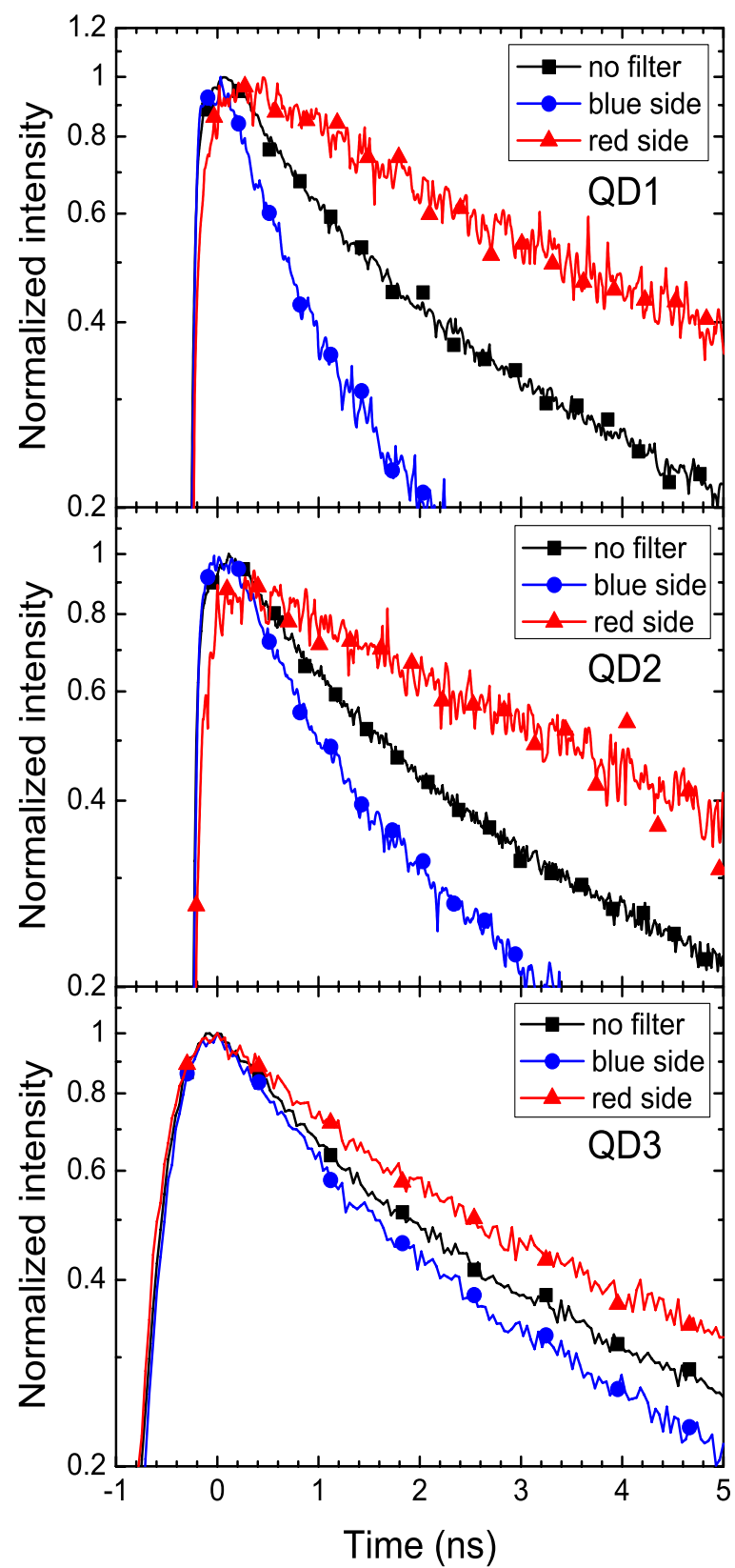

FIG. 3. (Color online) PL intensity decays of the overall ensemble emission (black line, squares) as well as for the blue side (blue line, circles) and the red side (red line, triangles) of the ensemble emission spectrum in layers with $c_{\mathrm{QD}} \approx 2.4 \times 10^{17} \mathrm{~m}^{-2}$ for the three samples studied (QD1-top, QD2-center, and QD3-bottom).

decay recorded from the whole ensemble (no filter) is shown in black (squares) and the decays on the blue and red sides of the QD emission spectrum are shown in blue (circles) and red (triangles), respectively. For all three QD samples a faster decay on the blue side and a slower decay on the red side with respect to the unfiltered decay is observed. Even at this one concentration there is evidence that the difference between the decay times on the blue and red sides decreases from QD1 to QD2 to QD3. This will be analyzed in more detail below. It is interesting to note that the decay on the red side of the QD emission spectrum shows a shift of the peak 
intensity to a later time, corresponding to a rise time of approximately $0.28 \mathrm{~ns}$ for the QD1 and QD2 layers. This is also consistent with energy transfer from the blue to the red side of the ensemble emission spectrum. However, there is no discernible rise time for QD3.

The time-dependent intensity decays $I(t)$ were fitted with a two-exponential decay function $I(t)=I_{1} \exp \left(-t / \tau_{1}\right)$ $+I_{2}\left(-t / \tau_{2}\right)$, where two decays times $\tau_{1}$ and $\tau_{2}$ with the respective intensity weights $I_{1}$ and $I_{2}$ were taken into account. The average lifetimes $\tau$ were calculated as intensity weighted means $\tau=\frac{I_{1} \tau_{1}^{2}+I_{2} \tau_{2}^{2}}{I_{1} \tau_{1}+I_{2} \tau_{2}}$. Average lifetimes $\tau_{\text {blue }}$ and $\tau_{\text {red }}$ have been determined for the decays on the blue and red sides of the PL emission spectrum for all QD layers. The average error on the extracted lifetimes is about $0.2-0.3$ ns. In Fig. 4, the average lifetimes of the blue side of the ensemble emission spectrum are shown for all three QD samples as a function of the QD concentration (solid squares, left-hand axis). The difference of the lifetimes from the blue to the red side, 1 $-\tau_{\text {blue }} / \tau_{\text {red }}$, is also shown (open circles, right-hand axis). For QD1 (Fig. 4, top panel) the lifetime on the blue side of the emission spectrum decreases from an initial value of about $9.0 \mathrm{~ns}$ at $0.4 \times 10^{17} \mathrm{~m}^{-2}$ to $2.2 \mathrm{~ns}$ at $3.4 \times 10^{17} \mathrm{~m}^{-2}$. The lifetime on the red side (data not shown here) is also decreasing with increasing concentration; however the influence of the QD concentration is less pronounced than for $\tau_{\text {blue }}$. In order to compare these two lifetimes over the range of concentrations investigated, the difference $1-\tau_{\text {blue }} / \tau_{\text {red }}$ is analyzed. For QD1 this difference is almost negligible at a concentration of $0.4 \times 10^{17} \mathrm{~m}^{-2}$ and increases to $75 \%$ at high concentrations. The concentration dependence of the lifetime difference follows a similar trend to the one observed for the FRET efficiency with increasing acceptor concentration in a mixed donor-acceptor QD monolayer. ${ }^{44}$ However, the values presented here cannot be understood as FRET efficiency as the lifetimes on the blue side of the ensemble emission spectrum are compared with the ones on the red side and not an undisturbed value of the blue side. The decrease in $\tau_{\text {blue }}$ is less pronounced for QD2 (Fig. 4, center panel) and the lifetime difference only increases to about $65 \%$ at high concentrations. For QD3 (Fig. 4, bottom panel) $\tau_{\text {blue }}$ shows an almost concentration-independent value of about $6.2 \mathrm{~ns}$ and $\tau_{\text {blue }}$ and $\tau_{\text {red }}$ show only a difference of $30 \%$ at high concentrations.

The decrease in $\tau_{\text {blue }}$ with increasing QD concentration (corresponding to a decreasing QD separation) confirms that the concentration effects presented here are due to energy transfer from the blue to the red side of the QD ensemble emission (from smaller to larger QDs) by the Förster mechanism, ${ }^{50,51}$ a nonradiative, resonant energy transfer process mediated by dipole-dipole interactions. In a monodispersed multilayer or three-dimensional QD structure the observed concentration effects on the optical properties of the QD structures will be even stronger. Comparing FRET between donor and acceptor QDs in a mixed QD monolayer and a separated donor/acceptor bilayer structure, slightly higher FRET efficiencies are expected for the mixed monolayer structure due to the smaller donor-acceptor separations in a closely packed layer. ${ }^{22}$ However, in the case of FRET in monodispersed QD systems, adding a second (or more) layer

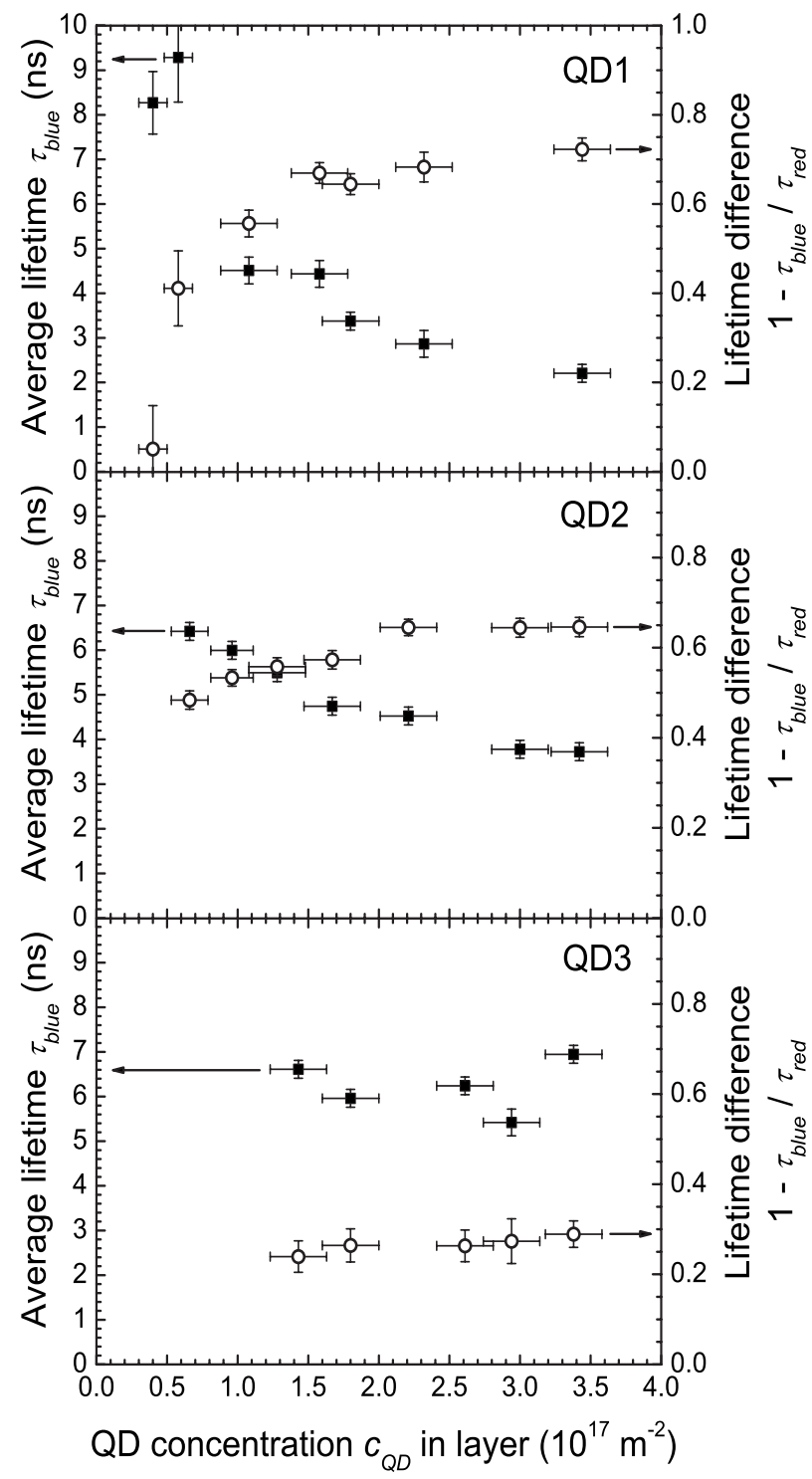

FIG. 4. QD concentration dependence of the average lifetime on the blue side of the ensemble emission spectrum (solid squares, left-hand axis) as well as of the lifetime difference between the red and blue sides (open circles, right-hand axis) for QD1 (top panel), QD2 (center panel), and QD3 (bottom panel) monolayers. The error on the average lifetimes extracted from two-exponential fits of the PL decays is approximately $0.2-0.3 \mathrm{~ns}$.

of QDs corresponds to an increase in the number of energy accepting QDs around a particular QD in the structure. Therefore, due to the increased QD concentration per unit volume, the probability for energy transfer within the ensemble will be enhanced. Increased energy transfer will lead to further shifts of the PL peak emission wavelength, a stronger decrease in the lifetime on the blue side of the ensemble emission spectrum and an increased difference in lifetime across the ensemble emission for three-dimensional QD structures.

\section{Theoretical modeling}

In this section the impact of intra-ensemble energy transfer on the lifetime in monodispersed QD layers will be in- 
vestigated in more detail. There are two nonradiative mechanisms for energy transfer from a donor to an acceptor which would both result in a decrease in the donor lifetime, the Dexter exchange mechanism ${ }^{52,53}$ and the Förster resonant energy transfer mechanism. ${ }^{50}$ The Dexter mechanism is concerned with excitation transfer via an exchange process requiring

the overlap of the wave functions between the donor and acceptor. It is a short-range mechanism, efficient typically on the subnanometer scale. ${ }^{52}$ In the QD monolayers the minimum distance that can be achieved between the donor and acceptor in a closely packed QD layer is given by the exclusion zone, $R_{e x}$, given by the sum of the radii of the QDs and the thickness of the TGA ligand shell of $0.5 \mathrm{~nm} .{ }^{54}$ The length of the ligand is only taken into account once, as the ligand shells can interpenetrate. ${ }^{19,54}$ This minimum separation of 2.7-3.2 nm for QD1, QD2, and QD3 will only be achieved at the highest concentrations and is still a relatively large separation in context of Dexter energy transfer. In contrast the Förster mechanism is less sensitive to the donoracceptor separation than the Dexter mechanism and can occur over longer separation distances. ${ }^{50,51}$ As discussed earlier there have been a number of previous theoretical and experimental studies showing that energy transfer between nanocrystal QDs can be appropriately described by FRET theory. ${ }^{18,21-23,40-43}$

In order to investigate the impact of intra-ensemble FRET on the lifetime in monodispersed QD layers in more detail, the lifetime decrease presented in Fig. 4 is compared to the concentration dependence of the lifetime predicted by a theory of FRET between randomly distributed donors and acceptors in two dimensions. ${ }^{47}$ This theory has previously been applied successfully to interpret the change in the donor PL decay as well as the acceptor concentration dependence of the FRET efficiency in a mixed donor-acceptor QD monolayer. ${ }^{44}$ In this theory the concentration dependence of the yield $q_{r}=\tau\left(c_{\mathrm{QD}}\right) / \tau^{0}$, the ratio of the concentrationdependent lifetime and an undisturbed value in the absence of energy transfer, is calculated according to Eq. (1) [Eq. (22) in Ref. 47] as a function of the Förster radius $R_{0}$ and an exclusion zone radius $R_{e x}$, that arises from the QD size as well as the thickness of the ligand shell.

$$
\begin{aligned}
q_{r}= & \int_{0}^{\infty} e^{-\lambda} \exp \left[-\pi C \gamma(2 / 3, \lambda / r) \lambda^{1 / 3}\right] \\
& \times \exp \left[\pi C r^{1 / 3}\left(1-e^{-\lambda / r}\right)\right] d \lambda .
\end{aligned}
$$

In Eq. (1) the incomplete Gamma function $\gamma(x, y)$ $=\int_{0}^{y} z^{x-1} e^{-z} d z$ is used; $r$ represents the ratio $R_{e x} / R_{0}$ and $C$ $=c_{\mathrm{QD}} R_{0}^{2}$ is a dimensionless concentration of the QDs. $R_{e x}$ is the exclusion radius, as described above. The Förster radius $R_{0}$ is the distance between a donor and an acceptor for which the FRET efficiency is $50 \%$.

The results of the modeling of the lifetime data based on the FRET theory in two dimensions are presented in Fig. 5. The values for $R_{e x}, R_{0}$, and the undisturbed lifetime $\tau_{\text {blue }}^{0}$ on the blue side of the QD ensemble emission spectrum used in the modeling of the lifetime decrease on the basis of Eq. (1) are given in Table II. The data is presented as the yield,

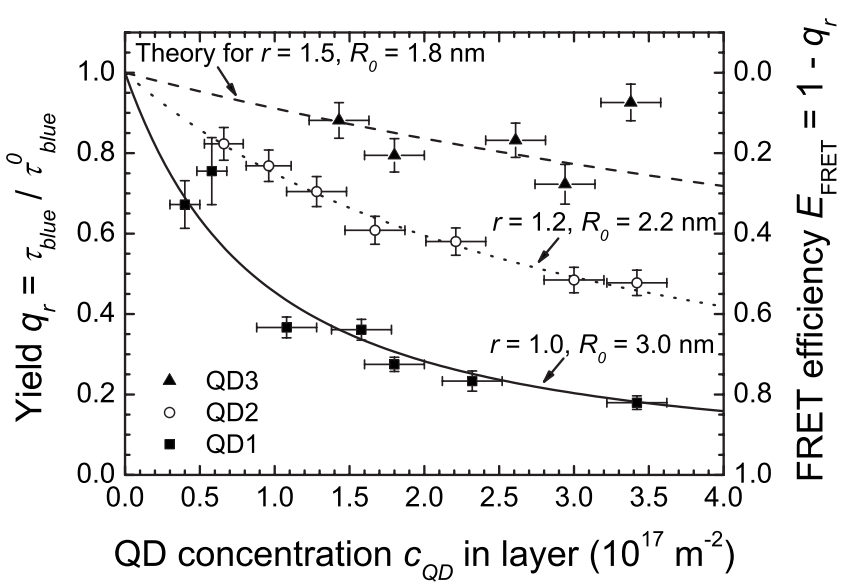

FIG. 5. Measured (symbols) and calculated (lines) lifetime yield $q_{r}$ (left-hand axis) in dependence of the QD concentration $c_{\mathrm{QD}}$ for samples QD1 (squares), QD2 (open circles), and QD3 (triangles). The corresponding values of the FRET efficiency $E_{\mathrm{FRET}}$ can be read from the right-hand axis. The estimated errors of the calculated yield and the FRET efficiency are approximately 5\%.

$q_{r}=\tau_{\text {blue }}\left(c_{\mathrm{QD}}\right) / \tau_{\text {blue }}^{0}$, as a function of $\mathrm{QD}$ concentration, where the data for the experimental yield $q_{r}$ is calculated by dividing the measured lifetime of each sample by the initial, undisturbed lifetime value $\tau_{\text {blue }}^{0}$ (given in Table II). It can be clearly seen that the yield decreases most strongly for QD1, less for QD2, and only little for QD3. The curves for the theoretical yield are calculated by numerical integration of Eq. (1). The optimum theoretical fits are represented as lines in Fig. 5. The value of $R_{e x}$ is essentially determined by the size of the QDs, which can be obtained from the spectral data. ${ }^{48}$ The values for the QD size and $R_{e x}$ determined in this way are given in Table II. Therefore, in fitting the experimental lifetime data $R_{e x}$ was kept within the error of $\pm 0.3 \mathrm{~nm}$ on the value extracted from the absorption spectra. As can be seen good agreement between the experiment and theory is obtained for each of the three types of QDs over the full range of concentrations investigated, indicating that the FRET mechanism can adequately explain the concentration dependence of the lifetimes measured in the monolayers. It can also be noted that no deviation from the FRET theory is observed at high concentrations for any of the three samples, indicating that even at these concentrations there is no evidence to suggest a transition to the Dexter energy transfer mechanism. Therefore it is concluded that FRET is the primary mechanism determining the concentration dependence of the optical properties of the monolayers.

Additionally, as can be seen in Fig. 5 (right-hand axis), control of the QD concentration provides a mechanism for tuning the FRET efficiency. For example, for QD1 the FRET efficiency, $E_{\mathrm{FRET}}=1-q_{r}$, can be varied from $0 \%$ to $85 \%$.

The Förster radius $R_{0}$ can also be calculated from the absorption and emission spectra of the QDs. Comparison of the $R_{0}$ values extracted from both the lifetime and spectral data provides a means to validate the parameters extracted from the lifetime data and confirm that FRET is the dominant mechanism influencing the QD concentration dependence of the PL lifetime of the QD layers. The Förster radius $R_{0}$ is described by 
TABLE II. QD size, exclusion zone radius $R_{e x}$ and Förster radius $R_{0}$ calculated from the absorption and emission spectra of the QD samples; and $R_{e x}, R_{0}$, and the undisturbed lifetime $\tau_{\text {blue }}^{0}$ extracted from theoretical modeling of the lifetime as a function of concentration. The error on the parameters is given in each column heading.

\begin{tabular}{|c|c|c|c|c|c|c|}
\hline \multirow[b]{2}{*}{ Sample } & \multicolumn{3}{|c|}{ Based on spectral QD properties } & \multicolumn{3}{|c|}{ Based on lifetime data } \\
\hline & Size $\underset{(\mathrm{nm})}{ \pm 0.2 \mathrm{~nm}}$ & $R_{e x} \underset{(\mathrm{nm})}{ \pm 0.3 \mathrm{~nm}}$ & $R_{0} \pm 0.2 \mathrm{~nm}$ & $R_{e x} \underset{(\mathrm{nm})}{0.3 \mathrm{~nm}}$ & $R_{0} \pm 0.2 \mathrm{~nm}$ & 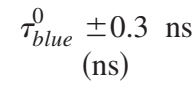 \\
\hline QD1 & 2.7 & 3.2 & 2.8 & 3.0 & 3.0 & 12.3 \\
\hline QD2 & 2.2 & 2.7 & 2.5 & 2.7 & 2.2 & 7.8 \\
\hline QD3 & 2.2 & 2.7 & 2.0 & 2.7 & 1.8 & 7.5 \\
\hline
\end{tabular}

$$
R_{0}=0.0211\left(\frac{\kappa^{2} Q_{D}}{n^{4}} J\right)^{1 / 6},
$$

where the orientation factor is given by $\kappa^{2}=2 / 3$ for randomly oriented dipoles, as is the case for the QDs in monolayers, and a volume-weighted refractive index $n=2.1$ is used. The quantum yield $Q_{D}$ is different for each QD sample and was determined by comparison with a Rhodamine $6 \mathrm{G}$ luminescence standard for the respective monolayers; it is in the range of 3-6\% for the three QD samples QD1, QD2, and QD3. The most important factor for the calculation of $R_{0}$ in this study is the spectral overlap $J$, which can be calculated using Eq. (3). ${ }^{51}$

$$
J=\int_{0}^{\infty} \hat{I}_{\mathrm{QD}}(\lambda) \cdot \varepsilon_{\mathrm{QD}}(\lambda) \cdot \lambda^{4} d \lambda,
$$

where $\hat{I}_{\mathrm{QD}}(\lambda)$ is the area-normalized emission spectrum and $\varepsilon_{\mathrm{QD}}(\lambda)$ is the extinction spectrum. The extinction spectrum is obtained from the absorption spectrum via Lambert-Beer's Law. ${ }^{44}$ Normally, in donor-acceptor systems, $J$ describes the overlap of the donor emission and the acceptor extinction spectrum. However, for the monodispersed QD structures investigated here Eq. (3) is used to calculate the overlap of the area-normalized emission spectrum $\hat{I}_{\mathrm{QD}}(\lambda)$ of a QD sample with its own extinction spectrum $\varepsilon_{\mathrm{QD}}(\lambda)$. $J$ will therefore be referred to as self-overlap in the following. The Förster radius $R_{0}$ determined from Eqs. (2) and (3) for the three QD samples are also summarized in Table II.

The values of $R_{e x}$ and $R_{0}$ obtained from the modeling of the concentration dependence of the lifetime yield $q_{r}$ agree well with the ones estimated from the spectral properties of the QD samples (see Table II). This unambiguously proves that the decrease in the lifetime with QD concentration is due to FRET from the blue to the red side of the QD ensemble emission spectrum. Furthermore it shows that the ligand shell sufficiently separates the QDs so that no other mechanisms such as surface effects due to direct contact or tunneling between the QDs has to be taken into account to interpret the lifetime data. These effects can therefore be neglected when studying layers formed with this type of chemically stabilized QDs.

As has already been reported for other monodispersed QD assemblies, the energy transfer is due to an overlap of the first absorption peak with the PL emission of the QDs.
This overlap, as calculated with Eq. (3), arises from the inhomogeneous broadening of the QD ensemble, represented by a large PL FWHM, and a small Stokes shift. ${ }^{16,18,20,23}$ The three samples can be characterized by the ratio of the PL FWHM and the Stokes shift. As this ratio decreases from $1.35(\mathrm{QD} 1)$ to $1.01(\mathrm{QD} 2)$ to $0.96(\mathrm{QD} 3)$, corresponding to a decrease in the overlap of the QD states, a reduced impact of the change in QD concentration on the optical properties of the QD monolayers is expected and observed. Increased intra-ensemble FRET in the QD1 and QD2 samples may also explain the sublinear increase in the integrated PL intensity, seen in Fig. 2, as energy losses might occur during the energy transfer.

\section{Impact of self-overlap}

In order to characterize the impact of FRET on the optical properties of all ten QD samples, the lifetime difference $1-\tau_{\text {blue }} / \tau_{\text {red }}$ is analyzed at a fixed concentration. Even though this parameter is not the real FRET efficiency $E_{\mathrm{FRET}}=1-q_{r}$, it has a similar concentration dependence as has been discussed above. The advantage of considering the lifetime difference, $1-\tau_{\text {blue }} / \tau_{\text {red }}$, is that it allows for evaluation of the probability of intra-ensemble FRET in a specific QD sample from a single measurement at only one concentration. Accurate determination of the FRET efficiency $E_{\text {FRET }}$ or the yield $q_{r}$ would require measurements on at least two monolayers of different concentrations, including one at a very low concentration. In Fig. 6 the lifetime difference as a function of the self-overlap $J$, calculated from the solution spectra with Eq. (3), is shown for all ten QD samples at a concentration $c_{\mathrm{QD}}$ of approximately $2.5 \times 10^{17} \mathrm{~m}^{-2}$. It can be clearly seen that the difference in lifetime of the blue and red sides of the ensemble emission increases with increasing self-overlap $J$, consistent with the stronger influence of intraensemble FRET on the optical properties of monolayers of QD samples with a higher self-overlap.

It is interesting to note that this trend can be fitted with the dependence of the FRET efficiency $E_{\mathrm{FRET}}$ on the self-overlap ${ }^{51}$

$$
E_{\mathrm{FRET}}=\frac{1}{1+\left[\sum_{i}\left(R_{0} / r_{i}\right)^{6}\right]^{-1}}=\frac{1}{1+\text { const. } \cdot J^{-1}} .
$$




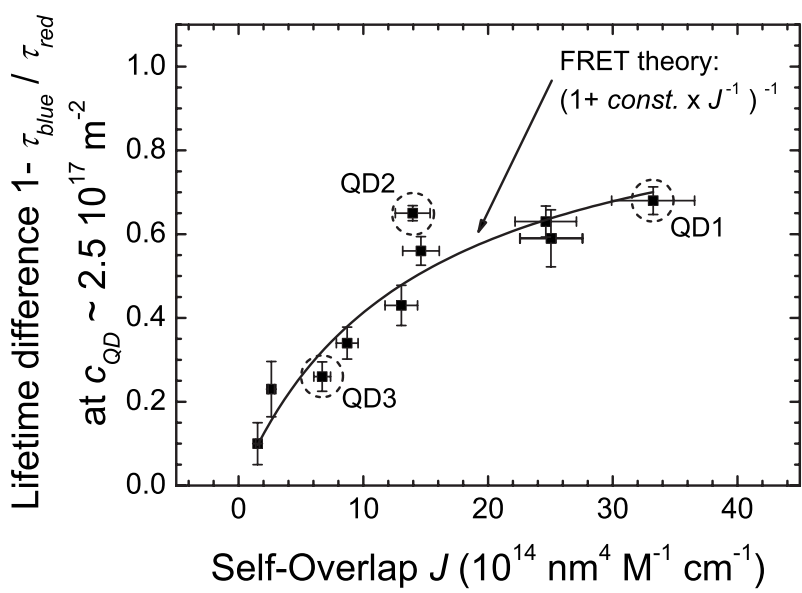

FIG. 6. Lifetime difference of the blue and red sides of the QD ensemble emission (squares) for monolayers, with a concentration $c_{\mathrm{QD}} \approx 2.5 \times 10^{17} \mathrm{~m}^{-2}$, as a function of the self-overlap $J$ for ten different QD samples. The theoretically calculated trend (line) is based on the dependence of the FRET efficiency on the self-overlap $J$.

The distance $r_{i}$ denotes the distance between one QD and its $i^{\text {th }}$ neighbor. In a closely packed layer six QDs are located in the first shell around a central QD. Assuming that only these nearest neighbors will be able to receive energy from the central QD due to a relatively large QD separation compared to $R_{0}$, the constant in Eq. (4) can be written as

$$
\text { const. }=\left(\frac{r_{D A}}{0.0211}\right)^{6} \frac{n^{4}}{6 \cdot \kappa^{2} Q_{D}} .
$$

As $J$ is calculated in units of $\mathrm{nm}^{4} \mathrm{M}^{-1} \mathrm{~cm}^{-1}$, the QD separation $r_{D A}$ has to be included in $\mathrm{nm}$ in Eq. (5). At a concentration $c_{\mathrm{QD}} \approx 2.5 \times 10^{17} \mathrm{~m}^{-2}$ closely packed layers are formed, and $r_{D A}$ will be approximately $R_{e x}$ which is an average $2.7 \mathrm{~nm}$ for the ten QD samples ranging in diameter from 1.0 to $2.8 \mathrm{~nm}$ and emission peak wavelengths between 511 and $553 \mathrm{~nm}$. The refractive index $n$ is 2.1 and the orientation factor $\kappa^{2}=2 / 3$ as stated above. Including a quantum yield $Q_{D}$ of $2 \%$ in the QD layer, the constant given by Eq. (5) is on the order of $1.1 \times 10^{15}$. The best fit, represented by a line in Fig. 6, is achieved for const. $=1.4 \times 10^{15}$, which is in reasonable agreement with the expected value taking into account the difference in size for the ten QD samples as well as variations in the quantum yield and QD concentration in the different monolayers. The good agreement between the trend of the FRET efficiency as a function of self-overlap with the lifetime difference indicates that it is reasonable to use the lifetime difference of the blue and red sides of the QD ensemble emission to indirectly observe the intra-ensemble FRET from the lifetime measurements at a single concentration.

For experimental studies of QD structures and devices, in particular those based on energy transfer, for light emitting diodes and photovoltaic application, it is important to be aware of the possibility of intra-ensemble FRET within the QD layers. Where intra-ensemble FRET could affect the results of measurements or impair device performance Fig. 6

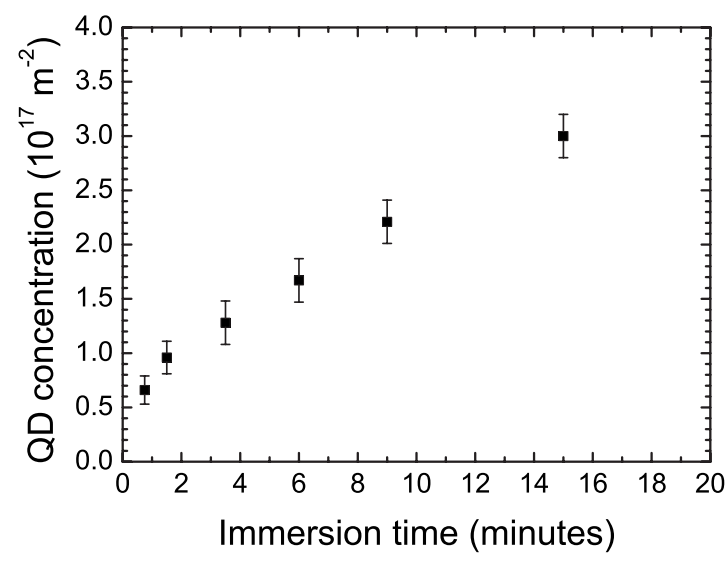

FIG. 7. Dependence of the quantum dot concentration in the layer-by-layer deposited layer on the immersion time.

indicates that QD samples with a self-overlap of less than $1.0 \times 10^{15} \mathrm{~nm}^{4} \mathrm{M}^{-1} \mathrm{~cm}^{-1}$ should be used for green emitting CdTe QDs. Equations (4) and (5) can be applied to determine the appropriate self-overlap for nanocrystal QDs of any material or size emitting in a different wavelength range. However, as the overlap depends on the Stokes shift and the FWHM of the PL peak, a small self-overlap is typically achieved at the expense of the quantum yield as the reduction in the FWHM is obtained by more stringent size selection, reducing the overall luminescence. Therefore, the need for a small self-overlap has often to be traded off against a loss in quantum yield.

\section{CONCLUSIONS}

The optical properties of QD monolayers have been investigated in detail for three QD samples of differing inhomogeneous broadenings and Stokes shifts. As the QD concentration in the layers increases the PL peak wavelength redshifts, the lifetime on the blue side of the PL emission spectrum decreases and the difference of the lifetimes of the QDs on the blue and red sides of the ensemble emission increases. These effects are attributed to Förster resonant energy transfer from smaller to larger QDs within the ensemble, facilitated by the overlap of the absorption and PL peak. The concentration dependence of the lifetime on the blue side of the QD ensemble emission can be reproduced by FRET theory in two dimensions, confirming that intraensemble FRET is the dominant process giving rise to the shortening of the QD decay with increasing concentration. For QD samples with a small self-overlap, due to a small PL FWHM and a large Stokes shift, the concentration effects are less pronounced. The deviation of the PL intensity from a linear increase with increasing QD concentration, in the samples in which the most energy transfer can occur, may be explained by energy losses due to increased energy transfer at high concentrations. The trend of an increasing influence of the QD concentration on the optical properties of QD monolayers for samples with increasing self-overlap is in good agreement with FRET theory. Temperature tuning for controllable FRET has been previously proposed. ${ }^{55}$ Here we 
have shown that it is possible to control the efficiency of the FRET process within the QD layers through variation in the QD concentration and/or careful selection of the inhomogeneous broadening and Stokes shift of the QD ensemble.

\section{ACKNOWLEDGMENT}

This work was financially supported by Science Foundation Ireland under Grant No. 05/PICA/1797.

\section{APPENDIX}

With a concentration of $1.5 \times 10^{-6} \mathrm{M}$, the QD solution for the layer-by-layer deposition of the QDs is not over saturated and for short immersion times in the range of tens of minutes, only submonolayer coverage is achieved. Therefore, the concentration of the QDs in the deposited layers can be varied by changing the immersion time as shown in Fig. 7.
*Author to whom correspondence should be addressed; bradlel@tcd.ie

${ }^{1}$ S. V. Gaponenko, Optical Properties of Semiconductor Nanocrystals (Cambridge University Press, Cambridge, 1998).

${ }^{2}$ A. R. Clapp, I. L. Medintz, and H. Mattoussi, ChemPhysChem 7, 47 (2006).

${ }^{3}$ U. Resch-Genger, M. Grabolle, S. Cavaliere-Jaricot, R. Nitschke, and T. Nann, Nat. Methods 5, 763 (2008).

${ }^{4}$ I. L. Medintz, A. R. Clapp, H. Mattoussi, E. R. Goldman, B. Fisher, and J. M. Mauro, Nat. Mater. 2, 630 (2003).

${ }^{5} \mathrm{M}$. Bruchez, M. Moronne, P. Gin, S. Weiss, and A. P. Alivisatos, Science 281, 2013 (1998).

${ }^{6}$ V. L. Colvin, M. C. Schlamp, and A. P. Alivisatos, Nature (London) 370, 354 (1994).

${ }^{7}$ K.-S. Cho, E. K. Lee, W.-J. Joo, E. Jang, T.-H. Kim, S. J. Lee, S.-J. Kwon, J. Y. Han, B.-K. Kim, B. L. Choi, and J. M. Kim, Nat. Photonics 3, 341 (2009).

${ }^{8}$ T. Rauch, M. Boberl, S. F. Tedde, J. Furst, M. V. Kovalenko, G. N. Hesser, U. Lemmer, W. Heiss, and O. Hayden, Nat. Photonics 3, 332 (2009).

${ }^{9}$ W. U. Huynh, J. J. Dittmer, and A. P. Alivisatos, Science 295, 2425 (2002).

${ }^{10}$ S. A. McDonald, G. Konstantatos, S. G. Zhang, P. W. Cyr, E. J. D. Klem, L. Levina, and E. H. Sargent, Nat. Mater. 4, 138 (2005).

${ }^{11}$ A. Chevreau, B. Phillips, B. G. Higgins, and S. H. Risbud, J. Mater. Chem. 6, 1643 (1996).

${ }^{12}$ F. Caruso, Colloids and Colloid Assemblies: Synthesis, Modification, Organization and Utilization of Colloid Particles (Wiley-VCH, Weinheim, 2004).

${ }^{13}$ A. L. Rogach, Semiconductor Nanocrystal Quantum Dots (Springer-Verlag, Berlin, 2008).

${ }^{14}$ G. Decher, Science 277, 1232 (1997).

${ }^{15}$ A. Shavel, N. Gaponik, and A. Eychmüller, Eur. J. Inorg. Chem. 2005 (18), 3613.

${ }^{16}$ M. Achermann, M. A. Petruska, S. A. Crooker, and V. I. Klimov, J. Phys. Chem. B 107, 13782 (2003).

${ }^{17}$ N. Cicek, S. Nizamoglu, T. Ozel, E. Mutlugun, D. U. Karatay, V. Lesnyak, T. Otto, N. Gaponik, A. Eychmüller, and H. V. Demir, Appl. Phys. Lett. 94, 061105 (2009).

${ }^{18}$ S. A. Crooker, J. A. Hollingsworth, S. Tretiak, and V. I. Klimov, Phys. Rev. Lett. 89, 186802 (2002).

${ }^{19}$ T. Franzl, D. S. Koktysh, T. A. Klar, A. L. Rogach, J. Feldmann, and N. Gaponik, Appl. Phys. Lett. 84, 2904 (2004).

${ }^{20}$ C. R. Kagan, C. B. Murray, and M. G. Bawendi, Phys. Rev. B 54, 8633 (1996).
${ }^{21}$ D. G. Kim, S. Okahara, M. Nakayama, and Y. G. Shim, Phys. Rev. B 78, 153301 (2008).

${ }^{22}$ M. Lunz, A. L. Bradley, W.-Y. Chen, and Y. K. Gun'ko, Superlattices Microstruct. 47, 98 (2010).

${ }^{23}$ S. F. Wuister, R. Koole, C. D. Donega, and A. Meijerink, J. Phys. Chem. B 109, 5504 (2005).

${ }^{24}$ C. Bertoni, D. Gallardo, S. Dunn, N. Gaponik, and A. Eychmüller, Appl. Phys. Lett. 90, 034107 (2007).

${ }^{25}$ M. Y. Gao, C. Lesser, S. Kirstein, H. Mohwald, A. L. Rogach, and H. Weller, J. Appl. Phys. 87, 2297 (2000).

${ }^{26}$ A. A. Mamedov, A. Belov, M. Giersig, N. N. Mamedova, and N. A. Kotov, J. Am. Chem. Soc. 123, 7738 (2001).

${ }^{27}$ J. Roither, S. Pichler, M. V. Kovalenko, W. Heiss, P. Feychuk, O. Panchuk, J. Allam, and B. N. Murdin, Appl. Phys. Lett. 89, 111120 (2006).

${ }^{28}$ J. De Girolamo, P. Reiss, M. Zagorska, R. De Bettignies, S. Bailly, J. Y. Mevellec, S. Lefrant, J. P. Travers, and A. Pron, Phys. Chem. Chem. Phys. 10, 4027 (2008).

${ }^{29}$ R. Kniprath, J. P. Rabe, J. T. McLeskey, D. Y. Wang, and S. Kirstein, Thin Solid Films 518, 295 (2009).

${ }^{30}$ Z. Q. Liang, K. L. Dzienis, J. Xu, and Q. Wang, Adv. Funct. Mater. 16, 542 (2006).

${ }^{31}$ C. A. Constantine, K. M. Gattas-Asfura, S. V. Mello, G. Crespo, V. Rastogi, T. C. Cheng, J. J. DeFrank, and R. M. Leblanc, Langmuir 19, 9863 (2003).

${ }^{32}$ G. de Bastida, F. J. Arregui, J. Goicoechea, and I. R. Matias, IEEE Sens. J. 6, 1378 (2006).

${ }^{33}$ C. C. Tu and L. Y. Lin, Appl. Phys. Lett. 93, 163107 (2008).

${ }^{34}$ Y. P. Rakovich, J. F. Donegan, M. Gerlach, A. L. Bradley, T. M. Connolly, J. J. Boland, N. Gaponik, and A. Rogach, Phys. Rev. A 70, 051801(R) (2004).

${ }^{35}$ V. Salgueiriño-Maceira, M. A. Correa-Duarte, M. Spasova, L. M. Liz-Marzán, and M. Farle, Adv. Funct. Mater. 16, 509 (2006).

${ }^{36}$ D. Y. Wang, A. L. Rogach, and F. Caruso, Nano Lett. 2, 857 (2002).

${ }^{37}$ Y. H. Chan, J. X. Chen, S. E. Wark, S. L. Skiles, D. H. Son, and J. D. Batteas, ACS Nano 3, 1735 (2009).

${ }^{38}$ V. K. Komarala, Y. P. Rakovich, A. L. Bradley, S. J. Byrne, Y. K. Gun'ko, N. Gaponik, and A. Eychmüller, Appl. Phys. Lett. 89, 253118 (2006)

${ }^{39}$ O. Kulakovich, N. Strekal, A. Yaroshevich, S. Maskevich, S. Gaponenko, I. Nabiev, U. Woggon, and M. Artemyev, Nano Lett. 2, 1449 (2002).

${ }^{40}$ G. D. Scholes and D. L. Andrews, Phys. Rev. B 72, 125331 (2005).

${ }^{41}$ G. Allan and C. Delerue, Phys. Rev. B 75, 195311 (2007). 
${ }^{42}$ R. Baer and E. Rabani, J. Chem. Phys. 128, 184710 (2008).

${ }^{43}$ C. Curutchet, A. Franceschetti, A. Zunger, and G. D. Scholes, J. Phys. Chem. C 112, 13336 (2008).

${ }^{44}$ M. Lunz, A. L. Bradley, W. Y. Chen, and Y. K. Gun'ko, J. Phys. Chem. C 113, 3084 (2009).

${ }^{45}$ S. J. Byrne, S. A. Corr, T. Y. Rakovich, Y. K. Gun'ko, Y. P. Rakovich, J. F. Donegan, S. Mitchell, and Y. Volkov, J. Mater. Chem. 16, 2896 (2006).

${ }^{46}$ A. L. Rogach, T. Franzl, T. A. Klar, J. Feldmann, N. Gaponik, V. Lesnyak, A. Shavel, A. Eychmüller, Y. P. Rakovich, and J. F. Donegan, J. Phys. Chem. C 111, 14628 (2007).

${ }^{47}$ P. K. Wolber and B. S. Hudson, Biophys. J. 28, 197 (1979).

${ }^{48}$ W. W. Yu, L. H. Qu, W. Z. Guo, and X. G. Peng, Chem. Mater.
15, 2854 (2003).

${ }^{49}$ R. S. Reilly, C. A. Smyth, Y. P. Rakovich, and E. M. McCabe, Nanotechnology 20, 095707 (2009).

${ }^{50}$ T. Förster, Ann. Phys. 2, 55 (1948).

${ }^{51}$ J. R. Lakowicz, Principles of Fluorescence Spectroscopy (Kluwer Academic/Plenum, New York, London, 1999).

${ }^{52}$ D. Dexter, J. Chem. Phys. 21, 836 (1953).

${ }^{53}$ M. Inokuti and F. Hirayama, J. Chem. Phys. 43, 1978 (1965).

${ }^{54}$ Z. Y. Tang, B. Ozturk, Y. Wang, and N. A. Kotov, J. Phys. Chem. B 108, 6927 (2004).

${ }^{55}$ R. Bose, J. F. McMillan, J. Gao, K. M. Rickey, C. J. Chen, D. V. Talapin, C. B. Murray, and C. W. Wong, Nano Lett. 8, 2006 (2008). 